How to cite this article:

Al-Megren, S., \& Almutairi, A. (2019). User requirement analysis of a mobile augmented reality application to support literacy development among children with hearing impairments. Journal of Information and Communication Technology, 18(2), 207-231.

\title{
USER REQUIREMENT ANALYSIS OF A MOBILE AUGMENTED REALITY APPLICATION TO SUPPORT LITERACY DEVELOPMENT AMONGST CHILDREN WITH HEARING IMPAIRMENTS
}

\author{
Shiroq Al-Megren \& Aziza Almutairi \\ King Saud University, Saudi Arabia \\ salmegren@ksu.edu.sa; aziza.m.m@hotmail.com
}

\begin{abstract}
Literacy is fundamental for children's growth and development, as it affects their educational, societal, and vocational progress. However, the mapping of language to printed text is different for children with hearing impairments. When reading, a hearingimpaired child maps text to sign language (SL) which is a visual language that can benefit from technological advancements, such as augmented reality (AR). Several research efforts that utilises AR for advancing the educational needs of people who are hearing impaired for different SLs are evident. Nevertheless, only a few directly elicit the visual needs of children who are hearing impaired. This study aims to address this gap in the literature with a series of user studies to elicit user requirements for the development of an AR application that supports the literacy development of Arab children who are hearing impaired. Three instruments were utilised in these user studies, each targeting a different group of literacy influencers: questionnaires issued to parents of children with hearing impairments, interviews with teachers, and observations of children who were deaf or hard of hearing. The findings indicated that the parents and teachers preferred Arabic SL (ArSL), pictures, and videos, whereas the children struggled with ArSL and preferred fingerspelling. These
\end{abstract}


preferences highlighted the importance of integrating various resources to strengthen written Arabic and ArSL literacy of Arab children. The findings have contributed to the literature on the preferences of Arab children who are hearing impaired, their educators, and their parents.

They also showed the importance of establishing requirements directly from intended disabled users in proactively supporting their learning process. The results of the study was used in the preliminary development of Word \& Sign, an AR mobile application intended to aid Arab children who are hearing impaired in their linguistic development.

Keywords: Arabic sign language, augmented reality, deaf and hard of hearing, hearing impaired, deaf reading, deaf education.

\section{INTRODUCTION}

It is critical to expose children to their first language within the first five years of their lives to allow them to acquire the necessary literacy skills (Mellon, et al., 2015). Literacy is vital to children's growth, as it notably affects their educational, vocational, and social development. Children naturally acquire a language vocally upon introduction to it, whereas reading requires language familiarity and decoding. Children grow up surrounded by conversation, and these interactions build familiarity with the language used. Decoding is a cognitive mapping process that is performed to associate vocal language with printed text (Goldin-Meadow, Mayberry, \& Read, 2001). Children with hearing impairments are often disadvantaged due to delayed exposure to their first language (i.e. sign language (SL) and a lack of phonemic awareness for decoding (Hanson, 1989).

Recent statistics from the General Authority for Statistics in Saudi Arabia has revealed that 76,902 individuals who are registered as deaf or hard of hearing (Kingdom of Saudi Arabia General Authority for Statistics, 2016) and share as their principal language-Arabic Sign Language (ArSL), which, like other SLs, is realised in the visual modality. This finding implies that when reading, a hearing-impaired person decodes printed text into visual forms, primarily identified as articulation (e.g., lip reading), fingerspelling, or SL (Treiman \& Hirsh-Pasek, 1983). Studies have found that SL is the most effective of 
the three visual forms to decode printed text and is the preferred option for hearing-impaired readers (Humphries \& MacDougall, 1999).

Various reading techniques are supported in the classroom to solidify the process of decoding printed text into SL, e.g., chaining and sandwiching (Humphries \& MacDougall, 1999). Both of these techniques consist of a series of associations presented by the teacher between a fingerspelled word and the same word in printed and SL forms. These visual approaches support the ability of children with hearing impairments to understand ideas represented visually in static or dynamic forms, i.e., visual literacy (Elkins, 2008). Current technologies can extend the printed text to the visual dimension and thus streamline and support a learning process tailored for such children. Augmented reality (AR) technology enriches the real world with virtual representations to enhance visual perception (Uluyol \& Sahin, 2016). Several benefits of the use of AR in education have been identified, including improved motivation and attention, smooth integration, accessibility, and creativity (Diegmann et al., 2015).

User-centred design (UCD) is a design process that focuses on the needs and limitations of users in the various stages of development. In UCD, users are actively involved in helping to formulate a clear understanding of user and task requirements. In this paper, we describe the initial work of designing a mobile AR application that utilises UCD to support the literacy development of children who are hearing impaired (deaf or hard of hearing). The requirements and visual needs of users for such an application came from a series of user studies. Teachers from deaf institutes and schools, children who were deaf or hard of hearing and parents of children who were hearing impaired participated in various quantitative and qualitative assessments. The findings demonstrated the importance of utilising various media to support the linguistic development of children when learning ArSL and Arabic, since preferences varied between the teachers, parents, and children. The results contributed toward developing Word \& Sign, a mobile AR application that is intended to aid the literacy development of children who are hearing impaired. The remainder of this paper is organised as follows. The related work section reviews work on the use of AR in the education of children with hearing impairments. It also presents the state of the art in AR development and evaluation. The following section describes the instruments used to elicit user requirements and identify the visual needs of such children. These instruments include interviews, observations, and questionnaires. Next, the results as described individually and cumulatively, followed by a presentation on the implementation of Word \& Sign. The final section concludes the paper and briefly summarises future directions of the research. 


\section{RELATED WORK}

This section reports on the state of the art in AR evaluation, highlighting several techniques and approaches. This section also reviews several works on AR applications intended for the educational development of children who are deaf or hard of hearing.

\section{Augmented Reality Evaluation Approaches}

The majority of the published literature on AR has focused on the development of new technologies and application prototypes, often intended for testing the potential of such technologies in various domains. However, few studies have addressed the needs and experiences of users or user-centric system evaluations. A literature survey conducted a decade ago showed that only $29 \%$ of published AR literature addressed the needs and experiences of users, and only some of those publications reported formal user evaluations (Dünser, Grasset, \& Billinghurst, 2008). Although the design process for AR systems applied UCD methodologies and techniques, user involvement is typically limited during the design phase of development. A survey of mobile AR systems and design approaches has highlighted questionnaires, interviews, and observations as some of the typical techniques used for AR assessment (Craig \& Craig, 2013). The remainder of this section reviews how techniques are utilised to support UCD for AR systems.

It is common to use questionnaires early in the design process to gain a better understanding of the needs of users and to elicit user requirements. Questionnaires have been utilised in various studies reported in the literature for AR systems (Correa, Tahira, \& Ribeir, 2012), as a first step toward design and implementation. Questionnaires have also been commonly utilised to assess the usability of AR prototypes at various levels of fidelity (Georgiou \& Kyza, 2017). However, typically teenagers or adults attempt questionnaires, i.e., users who can typically read, write, and comprehend questions. It is arguable whether this approach will prove inadequate for eliciting information from children. Questionnaires usually yield quantitative data that are systematically analysable.

Interviews entail formal oral conversations between potential or existing users and an interviewer. Unlike questionnaires, interviews yield qualitative data that require further analysis to extract relevant information. Analysis techniques for such qualitative data include thematic analysis, grounded theory, and discourse analysis (Turner, 2010). Similar to questionnaires, interviews have 
commonly been used to elicit requirements (Zainuddin, Zaman, \& Ahmad, 2009) and to assess the usability of various AR systems (O'Shea, Dede, \& Cherian, 2011). Typically, qualitative data regarding such systems are subject to thematical analysis to understand users' ideas, thoughts, and feelings, better.

Another research method that is commonly utilised in AR design is user observation. In this approach, an observer monitors users within the context of their work or home life. These observations are usually video-recorded and later analysed to derive a set of functional requirements, assess usability, or evaluate system performance. For instance, observational studies were conducted before the development of a mobile AR application for in-store shopping to gain an understanding of the role played by mobile devices in the shopping experience (Xu, Spasojevic, Gao, \& Jacob, 2008). User observation studies have also been conducted to extract holistic findings for qualitative or quantitative assessment of the behaviour of users when interacting with AR prototypes (Yilmaz, 2016).

\section{Augmented Reality and Education for the Hearing Impaired}

The use of technology to enhance literacy and linguistic ability is greatly encouraged in the literature, (Samuel \& Zaitun, 2007). AR is a relatively low-cost innovation easily utilised in educational settings. The potential of AR for enhancing learning and teaching has been identified in various works (Billinghurst \& Dünser, 2012; Diegmann et al., 2015; Dunleavy, Dede, \& Mitchell, 2009; Garrett, Jackson, \& Wilson, 2015). Recent technological advances in mobile computing have also made it possible in rapidly developing mobile AR systems. In an educational setting, AR can be used as a cognitive tool that places the learner within a real-world physical and social context while still facilitating participatory and metacognitive learning (Dunleavy et al., 2009; Sungkur, Panchoo, \& Bhoyroo, 2016). AR in education supports interactive learning, simplicity, contextual information acquisition, efficiency and effectiveness, interpretation skills, and creativity (Sungkur et al., 2016).

Despite various reports in the literature highlighting the value of $A R$ as an educational tool, few works have addressed the development of AR systems for physically and/or mentally disabled children. Due to its relevance to the present study, the remainder of this section reviews the literature on utilising AR and SL for the education of learners (children and/or adults) who are hearing impaired. Table 1 summarises reviewed works from the perspectives of user involvement and evaluation. 


\section{Table 1}

Summary of User Methods and Techniques Applied with Regard to Reviewed AR Applications for Learners who are Hearing Impaired.

\begin{tabular}{ccccc}
\hline User Case & SL & $\begin{array}{c}\text { Hearing-impaired } \\
\text { Learners }\end{array}$ & $\begin{array}{c}\text { Educational } \\
\text { Domain }\end{array}$ & $\begin{array}{c}\text { Requirement } \\
\text { Gathering }\end{array}$ \\
\hline $\begin{array}{c}\text { Zainuddin, Zaman, } \\
\text { \& Ahmad, 2011 } \\
\text { and } \\
\text { American }\end{array}$ & $\begin{array}{c}\text { Malaysian } \\
\text { children }\end{array}$ & $\begin{array}{c}\text { Elementary school } \\
\text { Science }\end{array}$ & $\begin{array}{c}\text { Interviews and } \\
\text { observations }\end{array}$ \\
$\begin{array}{c}\text { Oka Sudana, } \\
\text { Aristamy, \& }\end{array}$ & Indonesian & Children or adults & Literacy & Not reported \\
$\begin{array}{c}\text { Cadenanes \& } \\
\text { Arrieta, 2014 }\end{array}$ & American & Children & Sign & Not reported \\
Kožuh, Hauptman, \\
Kosec, \& Debevc, \\
2015
\end{tabular}

Preliminary investigations were conducted to determine the needs of Malaysian science students with hearing impairments with the goal of enhancing existing courseware (Zainuddin et al., 2009; Ahmad, Nooraishya \& Ali, 2018). It involved identifying three students observed in various tasks, and had common characteristics, the main characteristic being that the hearing-impaired students were visual learners and had more difficulty recognising text than pictures. Interviews with education officers helped to further investigate the difficulties facing the hearing-impaired science learners. These explorations were used to guide the development of PekAr-Mikroogranisma, a science courseware package that utilises a web-based AR application (Zainuddin, Zaman, \& Ahmad, 2011). The courseware augmented with three-dimensional (3D) models of organisms via AR, supports visual descriptions expressed 
in Malaysian SL (MySL). Heuristic evaluations evaluated the application in terms of interface usability, pedagogy, AR environment, and video SL. Although the feedback was largely positive, several suggestions put forward contributed to guide future improvements.

An AR mobile application was developed to provide 3D representations of Indonesian SL (Oka Sudana, Aristamy, \& Wirdiani, 2016). The application recognises flashcards of printed letters and augments the flashcards with the corresponding SL fingerspelling signs. The flashcards can assemble into words, and the application then fingerspells each of the letters through animation, which is open to manipulation to explore the signing of the letters from various angles. Although the intended application is for SL learners who are not hearing impaired, its benefits can extend to hearing-impaired visual learners.

AR technology was used to develop the Multilanguage Cycle (MuCy) model, an SL teaching model (SLTM), to supplement SL pedagogical materials (SLPMs) with interactive visual content (Cadenanes \& Arrieta, 2014). The model supports two levels of teaching for communication in American SL (ASL) and aims to assist the development of teachers of students with hearing impairments. The first level establishes connections between ASL, visual references, and printed text. Vocalising the printed text is at the second level, and the SLPMs (i.e., the materials input for AR) augmented with avatars utilising ASL. An experimental study conducted found that the use of SLPMs resulted in a significant improvement in communication skills. MuCy provides teachers with a complementary and adaptable SLTM that promotes the comprehension of concepts required for teaching hearing-impaired children.

For Slovenian SL, an AR application was developed and evaluated to determine the efficiency of using an AR application when learning SL (Kožuh, Hauptman, Kosec, \& Debevc, 2015). The application detects an image of an SL interpreter and augments it with a video of the interpreter signing the word. In an experimental study, there was a comparison in performance of the AR application against SL images and live SL from an SL interpreter. The application proved superior, as compared with images of SL, with users performing better with the augmented interpretations. However, the application did not fare as well as a live SL interpreter. Thus, the authors of the study recommend the use of their AR application when teaching SL as a complementary educational tool in combination with current practices.

Najeeb is a mobile AR application developed to educate children who are hearing impaired on Islamic religious traditions (Alnafjan, Aljumaah, 
Alaskar, \& Alshraihi, 2017). Before the development of Najeeb, a review of the literature conducted identified a need for educational resources for Arab children who are deaf or hard of hearing. Najeeb uses images, videos, and 3D animations to support the teaching of the basic tenets of Islam. The design of Najeeb grounded in a theoretical framework of information processing theory promotes engagement and learning in e-learning environments. The developers of Najeeb have not reported on the design process or the level of user involvement.

There are several existing mobile applications that are intended to support learners of Arabic and ArSL, such as Al-Alturjuman (Mind Rockets Inc., 2017), Hayaak (mHealth Company, 2016), and Tawasoly (Al-Nafjan, AlArifi, \& Al-Wabil, 2015). However, none of them utilises AR; moreover, their development processes are largely unreported, and thus, the level of user involvement in collecting information to be translated into clear user needs remains unknown. This is also the case for Najeeb (Alnafjan et al., 2017), for which no formal methods were utilised to identify user needs and experiences. This situation prevails not only for AR applications for ArSL but also for AR systems designed for various other SLs (see Table 1). This gap in the development of AR systems highlights the value of the current work, in which the aim is to identify the needs and experiences of users before system development using formal research methods that support UCD.

\section{RESEARCH METHODOLOGY}

This study is part of a research effort that aims to develop an AR application as a tool to advance the literacy skills of Arab children who are deaf or hard of hearing. Adopting a UCD approach for the development necessitates the active involvement of the intended users to formulate a clear understanding of user needs. This process is particularly imperative for user needs that do not match those of typical non-disabled users. For this purpose, before development, there was a series of studies conducted to determine the visual needs of Arab children who were hearing impaired as they learned new vocabulary including how to read. In this paper, the elicitation of user requirements began with gathering information from the parents of children who were deaf or hard of hearing, followed by interviewing special needs educators, and finally observing children who were hearing impaired as they associated printed text with visual media.

There are several research methods that are commonly utilised for eliciting user requirements, i.e., interviews, focus groups, questionnaires, and observations. 
A survey of the literature identified typical research methods utilised for mobile AR system design and development, which include questionnaires, interviews, and observations (Craig \& Craig, 2013). Questionnaires are popularly utilised in the AR literature (Correa et al., 2012; Georgiou \& Kyza, 2017) at the various stages of development. This approach is easy to use, low- or no-cost, and can reach a large number of respondents. However, questionnaires have certain disadvantages, such as respondents arbitrarily selecting answers and their inability to express their thoughts with the options provided. Carefully designed questionnaires and an approach that uses both quantitative and qualitative questions help overcome these drawbacks. Interviews are commonly utilised early in the development phase and used regularly in the development of AR mobile systems (Zainuddin et al., 2009; O'Shea et al., 2011). This approach follows a structured methodology wherein topics are determined a priori and transcripts are prepared at the end of an interview for analysis. Unlike questionnaires, user interviews allow for more expressiveness, and the findings are often thematically analysed. Nevertheless, a drawback of interviews is users' expressions of their needs or actions do not always reflect their actual needs or actions. Questionnaires and interviews provide valuable user feedback, dictated by a set of questions, but direct observations are at times better suited for requirement gathering. Observations allow researchers to observe users in their current environment and examine the various elements that affect their interaction with a system. Several disadvantages can inhibit researchers from understanding the users' actual requirements, such as bias or unnatural behaviour of users-the result of being under observation.

The user requirements in the elicitation stage of this research included three studies to determine the visual needs of Arab children who were hearing impaired from the children themselves, parents, and educators. In the first user study, parents responded to a questionnaire to understand the literacy needs of their children who were hearing impaired. The choice of the questionnaire as a research instrument to use with the parents was due to the sensitivity of the topic in Arab culture, where parents might feel more comfortable discussing their children's issues from behind a barrier, i.e., a computer screen. A more rigorous instrument, interviews, was used with educators of children who were hearing impaired in the second user study. The interviews were semistructured to allow the interviewer to explore subjects related to the context of the study. The educators were more aware as compared with the parents on the educational needs of children who were hearing impaired due to their experience in addressing the visual needs of these children in an educational setting. Therefore, interviews were more appropriate for the educators in the expression of the educational requirements of the children who were hearing impaired. In the third and final user study, observations assisted in fully 
grasping how the intended users (i.e., the children who were hearing impaired) react to the different visual media prescribed by their parents and teachers.

\section{USER REQUIREMENT ELICITATION}

Qualitative assessments conducted helped to determine the visual needs of children who were hearing impaired while learning to read. There were three groups of influencers considered for eliciting user requirements: hearing-impaired children, their parents, and their teachers. There was an appropriate instrument used for each group: observations, questionnaires, and interviews, respectively. The remainder of this section reports on the research methodology applied for each of the utilised instruments in the order applied, starting with questionnaires, followed by interviews, and ending with observations.

\section{User Study 1}

Parents play an important role in the literacy development of their children. In the case of children who are hearing impaired, studies have revealed delays in literacy for those born to hearing parents because of the associated delays in the children's exposure to SL (Alyami, Soer, Swanepoel, \& Pottas, 2016; Geeslin, 2008). Due to the significance of parental influence, there was a questionnaire designed to identify patterns relating to the literacy of the children who were hearing impaired from the parents' perspective. The use of the questionnaire was also to understand better the different learning dynamics at school and at home. As a research instrument, the choice of a questionnaire-based initial approach was due to its ease of use and the opportunity it provided for standardisation. The sensitivity of the topic considering the cultural view of disability was also one of the primary reasons for using questionnaires instead of the more rigorous interviews.

\section{Participants}

Fourteen parents of children who were hearing impaired responded to the questionnaire. Demographic information was collected about the parents, including their gender (10 mothers and 4 fathers), age (average age of 43 years, with a standard deviation of 5.3 years), and level of education (the majority, 87\%, were educated at university). Recruitment of the respondents was via various platforms, mainly Facebook, Twitter, and WhatsApp.

\section{Materials}

The design of the questionnaire focused on investigating the needs of hearingimpaired children from the perspective of their parents. It consisted of a 
combination of open- and closed-ended questions for collecting information related to the children's literacy development (reading and ArSL). The questionnaire consisted of the following four main sections.

- General information: This section gathers information pertaining to the child's age, gender, hearing loss range, grade in school, and scholastic performance.

- Reading and ArSL: In this section, the questions posed gather information about the reading skills and ArSL proficiency of the child.

- Technology: This section prompts the parent for more details about his or her child's technical skills, competence, and preferences.

- Recommendation: The final section asks the parent to provide recommendations based on his or her child's visual needs and technical preferences.

\section{Procedure}

The distribution of the questionnaire was via various online platforms, including Facebook, an online social group for people who have hearing impairment, Twitter, and WhatsApp. Participants can only complete the questionnaire once to avoid result duplication; and confirmation was by registering the email address of each respondent. Participants were required to answer all questions, and they were encouraged to answer without any bias. The questionnaire was open to response for three weeks, before closing for analysis.

\section{Results}

Demographic information about the children was the first to be collected. Among the children, 57\% and $43 \%$ were male and female, respectively, with ages ranging from seven to 17 years. Of these children, $50 \%$ were born with hearing impairments, 36\% lost their hearing before two years of age, and $14 \%$ lost their hearing after they turned two years old. For the majority of the children, their hearing loss was diagnosed as profound; 29\%, as severe; and $14 \%$, as moderate. The questionnaire also prompted the parents to discuss their children's performance in school; $57 \%$ reported good performance, $14 \%$ reported very good performance, and the remainder reported poor performance.

After completing the demographic section of the questionnaire, the parents responded to questions on their children's reading skills and SL proficiency. Almost two-thirds of the parents stated that their children used ArSL, whereas 
the remaining parents claimed that their children did not know ArSL or any other SL. The children in the latter category ranged from 10 to 15 years old and all were born with hearing impairments. The parents also assessed the reading levels of their children. The results showed that $36 \%$ of the children were good in reading, $36 \%$ were poor readers, and $29 \%$ did not know how to read. To gain a better understanding of the main influencers of their children's reading and ArSL development, the parents identified the main resources they used for this purpose. The children's families and educational institutions for the hearing impaired, followed by inclusive classes and self-learning primarily influenced both the reading and ArSL skills. The parents listed some of the difficulties encountered by their children. These difficulties included weak exposure to rich linguistic content, immature decoding skills, unfamiliarity with language (i.e., ArSL), and difficulties in comprehension.

With regard to the use of technology, it was important to understand the types of technologies and applications often used at home. All the parents stated that their children had used smartphones and/or tablets in the past. The majority of the children mainly used such technologies for entertainment, and only $42 \%$ used them for educational purposes. The children interacted with the visual representations provided by these technologies, and the parents gave their opinion regarding what attracted their children to an application(s). Videos proved to be the most popular, followed by pictures, animation, ArSL, and fingerspelling.

The final section of the questionnaire prompted the parents to rank different types of visual representations to provide recommendations for an AR application to support the acquisition of reading skills. The parents ranked pictures and videos as the most strongly preferred methods of representation in their responses, followed by SL and fingerspelling.

\section{User Study 2}

Interviews conducted with educators (elementary school teachers) probed their experiences, attitudes, and desire in order to gain a better understanding of the visual needs of their hearing-impaired students. Generally, interviews were common for similar purposes. A semi-structured approach was used to enable a flexible exploration of the subject matter and further elucidation when necessary to elicit user requirements (McIntosh \& Morse, 2015).

\section{Participants}

Seven female teachers recruited for interviews were from the Al-Amal Institute for the Deaf and the 300th Public Elementary School of Riyadh. The teachers 
had a mean age of 39.57 years (with a standard deviation of $\mathrm{SD}=8.62$ years) and taught children at the elementary level. The teachers had an average of 15.57 years of experience in teaching children with hearing impairments, with a standard deviation of 8.98 years. Three of the teachers taught comprehension and reading, two taught math, one taught art, and the other taught science.

\section{Materials}

A devised interview protocol served as a guide for the interviewer to ensure that topics covered were pertinent to the research, which resulted in a schematic presentation of questions/topics to be explored pertaining to teaching strategies and difficulties encountered by children who were deaf or hard of hearing as well as the visual representations of textual information. The interviews addressed the following topics:

- $\quad$ Current practices adopted by teachers and interpreters when working with hearing-impaired children, such as the use of technology or teaching methods and techniques, as well as difficulties encountered during teaching.

- Visual representations of text utilised during lessons or for interpretation. This part of the interview investigated the preferred media for visual learning from the perspective of the teachers.

- Demonstration of two AR applications, Magic Camera (Baby Sign and Learn, 2013) and Anatomy 3D (DAQRI, 2015), to clarify the idea behind the research.

\section{Procedure}

All interviews began with an introduction and presentation of the research goals and objectives. All the interviewees gave their informed consent to participate in the study. Next, the teachers provided information about their age and teaching experience. Then, the interviewer proceeded with asking questions regarding the visual needs of students who were hard of hearing or deaf. When necessary, the interviewees elaborated to contextualise their answers during the interview. At the end of each session and after questions were concluded, recommendations were collected from the interviewees regarding AR input, required support, and methods for focusing attention and learning. The interviews spanned two days including several sessions at the interviewees' respective workplaces. Each interview session conducted individually in Arabic lasted approximately an hour. All sessions were audiorecorded. 


\section{Results}

The transcribed and then translated interviews were in English. Thematic analysis was used to identify, analyse, and report on themes found within the transcripts (Braun \& Clarke, 2006). Thematic analysis is a flexible analysis approach that is used to examine the perspectives of participants and to highlight similarities and differences to generate insights (Nowell, Norris, White, \& Moules, 2017). This approach is commonly used in the literature to elicit user requirements from participants regarding various technologies, including AR applications (Pinto, et al., 2017; Zhu, Hadadgar, Masiello, \& Zary, 2014).

\section{Table 2}

Thematic Analysis Protocol Utilised to Extract Results From Interview Transcripts

\begin{tabular}{cl}
\hline Protocol Steps & \multicolumn{1}{c}{ Descriptions } \\
\hline 1 & $\begin{array}{l}\text { Read transcripts to familiarise with data. Typically, the analyst notes } \\
\text { initial ideas in this step. }\end{array}$ \\
2 & $\begin{array}{l}\text { Code and collate important features that appear interesting or } \\
\text { meaningful. Form candidate themes by combining or splitting } \\
\\
\end{array}$ \\
& collated codes. \\
& Review and refine candidate themes and define their scope. This \\
& step results in the definition and naming of high-level themes. \\
& Final contextualisation of findings.
\end{tabular}

Table 2 describes the protocol used to extract results from the interviews. The findings were as follows:

- $\quad$ Visual representations of text: The teachers advocated SL as the main visual representation to introduce vocabulary and clarify concepts. However, there was a recommendation that SL combine with pictures and/or videos. There was an equal division in the teachers' beliefs when asked for their recommendations regarding the use of avatars, real images, or videos. Some of the teachers recommended avatars because they believed that younger children would find them more enjoyable than real images or videos.

- Vocabulary: The interviews addressed several language difficulties, including the identification of words in context or their connotations, 
synonyms and antonyms, and adjectives and adverbs. The teachers also noted that the children struggled with grammar.

- $\quad$ Linguistic exposure: The majority of the teachers confirmed indications of reduced linguistic skills amongst hearing-impaired children compared with others. They advised enriching the linguistic content introduced to these children to compensate for their disability and consequent isolation.

- $\quad S L$ : SL was the main means of communication with the hearingimpaired children. Children raised in a hearing household particularly encountered difficulties with SL.

- Technical resources: The teachers often used various means of displaying pictures and videos to convey the meanings of words taught in a lesson. One teacher reported that she utilised an AR application to aid in speech and pronunciation. She stated that it was easier to use than a projector because her lessons were individualised.

- $\quad A R$ input: The teachers recommended the use of printed books for elementary-grade children with hearing impairments because of their ease of maintenance compared with that of flashcards. The teachers also recommended an electronic version to involve parents in the teaching process.

\section{User Study 3}

Observations of users of AR systems are commonly utilised as a means of monitoring users within the user context to identify patterns of activity and general user concerns (Barma, Daniel, Bacon, Gingras, \& Fortin, 2015; Yilmaz, 2016). Assessment of the performance of the children with regard to various visual media: videos, pictures, ArSL, and fingerspelling via controlled formal observations helped to determine the visual needs of the children who were deaf or hard of hearing. There was systematic coding and classifying of observed behaviours using a controlled approach based on association tasks.

\section{Participants}

Fourteen children from first-, second-, and fifth-grade were recruited from Al-Amal Institute for the Deaf (six children) and the 300th Public Elementary School of Riyadh (eight children). Seven children were from the first grade (average age $=8.71$ years, $\mathrm{SD}=2.06$ ), four children were from the second grade (all nine years old), and three children were from the fifth grade (average age $=14$ years, $\mathrm{SD}=3.46$ ). All children were female and had moderate to severe hearing loss. None of the children suffered from any other disability. 


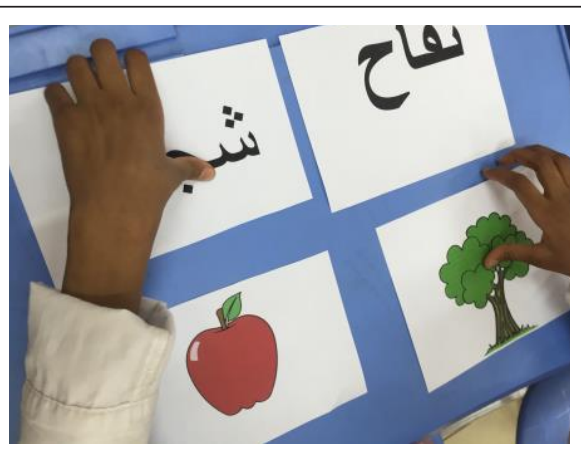

Figure 1. A child who is deaf completing the text-to-picture association task.

\section{Materials}

A set of four association tasks in visual media (videos, pictures, ArSL, and fingerspelling) presented in the observation sessions to the children encouraged their participation and permitted an objective assessment of their performance. For the various media, both realistic and cartoon representations presented to the children assisted in identifying their preferences for realistic or animated representations. Each task involved a series of associations between the printed text and the main visual medium considered. The children associated printed text with videos, pictures, ArSL, or fingerspelling for each task. The selection of text used in the tasks came from the original courseware for the elementary school children, and the words used included only those covered in their lessons. Sixteen different words making up 16 sub tasks chosen helped to reduce the effects of learning and practice. The tasks were as follows:

Associating text with pictures: Text and pictures were printed and spread out in an arbitrary configuration on a table. The children associated the textual samples with the correct pictures. Figure 1 shows some of the words and images used for the text-to-picture association task. Four sub tasks were used, two realistic pictures and two cartoon pictures.

- $\quad$ Associating text with videos: The children looked at printed textual samples distributed on a table, and then they watched a video on a smartphone. Next, the children associated the content of the video with the correct text. Similar to the previous task, four sub tasks were utilised, two representing realistic videos and two showing cartoon representations of words.

- $\quad$ Associating text with ArSL: In this task, the children looked at textual samples and watched videos of ArSL. After each video, the children 
associated the observed signs with the correct words. This task was also used to investigate the children's preferences regarding full-body or hand-focused interpretations. This task was made up of five sub tasks: two sub tasks showed a human interpreter, another two showed an animated interpreter (i.e., an avatar), and one sub task showed only the interpreter's hands.

- $\quad$ Associating text with fingerspelling: For the final task, the children associated each fingerspelt picture with the corresponding word from textual samples and fingerspelt pictures (pictures of ArSL letters) spread out in a random configuration on a table. This association task consisted of three sub tasks.

\section{Procedure}

At the beginning of each session, the observer introduced herself to the children, while the teacher interpreted the communication. Next, each child gave general information regarding her age and degree of hearing loss. Then the children performed tasks, individually. In each task, the children correctly associated each piece of media with its corresponding text. Each child had two opportunities. After the second incorrect answer, the teacher interpreted the printed text using ArSL. After completing the tasks, the children were introduced to AR using two applications: Magic Camera (Baby Sign and Learn, 2013), an ASL application designed to teach toddlers new words, and Let's Read (Kids App, 2017), an application that interprets stories using ArSL, sounds, and images. The observation sessions were conducted at the children's own schools, as recommended in the literature (Mich, 2009), and were spread over four sessions. Children from the first- and fifth-grade attended two sessions at Al-Amal Institute and children from the first- and second-grade attended the other two sessions conducted at the public school. Each session lasted approximately two hours with the observations documented in text and photographs.

\section{Results}

Table 3 summarizes the observation results regarding the performance of the 14 children in the association tasks. As expected the children from fifthgrade performed better than, the children from first- and second-grade. The following discusses the children's performance relative to the type of task, namely, text-to-picture, text-to-video, text-to-SL, or text-to-fingerspelling association.

Text-to-picture association: All three of the children from fifth-grade were able to complete this task correctly. The children from first-grade performed the worst as they often made mistakes when working with cartoon pictures. 
In fact, only one child from the first grade completed the task without any mistakes. The children from second-grade fared slightly better but did not perform as well as the children from fifth-grade.

Text-to-video association: The children from second- and fifth-grade performed equally well in this task, with most of the children completing the task correctly for all four videos. The children from fifth-grade made mistakes when considering realistic videos, whereas in the case of the children from second-grade, the mistakes occurred when associating cartoon videos with text.

Text-to-SL association: Although all of the children struggled with ArSL, the children from fifth-grade performed slightly better than, children from the first- and second-grade. Their struggle was more evident with an animated interpreter rather than with a human interpreter.

Table 3

Task Performance of Hearing-impaired Children in all Four Sessions

\begin{tabular}{|c|c|c|c|c|c|c|c|c|c|c|c|c|c|c|}
\hline \multirow[t]{2}{*}{ Task } & \multicolumn{7}{|c|}{ First Grade } & \multicolumn{4}{|c|}{ Second Grade } & \multicolumn{3}{|c|}{ Fifth Grade } \\
\hline & S1 & $\mathrm{S} 2$ & S3 & $\mathrm{S} 4$ & S5 & S6 & S7 & S8 & S9 & $\mathrm{S} 10$ & S11 & $\mathrm{S} 12$ & $\mathrm{~S} 13$ & S14 \\
\hline Realistic picture 1 & $\checkmark$ & $\checkmark$ & $\checkmark$ & $\checkmark$ & $\checkmark$ & $\checkmark$ & $\checkmark$ & $\checkmark$ & $\checkmark$ & $\checkmark$ & $\checkmark$ & $\checkmark$ & $\checkmark$ & $\checkmark$ \\
\hline Realistic picture 2 & $\checkmark$ & $\checkmark$ & $x$ & $\checkmark$ & $\checkmark$ & $\checkmark$ & $\checkmark$ & $\checkmark$ & $x$ & $\checkmark$ & $\checkmark$ & $\checkmark$ & $\checkmark$ & $\checkmark$ \\
\hline Cartoon picture 1 & $x$ & $\checkmark$ & $x$ & $\checkmark$ & $x$ & $x$ & $x$ & $\checkmark$ & $x$ & $\checkmark$ & $\checkmark$ & $\checkmark$ & $\checkmark$ & $\checkmark$ \\
\hline Cartoon picture 2 & $\checkmark$ & $\checkmark$ & $x$ & $\checkmark$ & $\checkmark$ & $x$ & $\checkmark$ & $\checkmark$ & $\checkmark$ & $\checkmark$ & $\checkmark$ & $\checkmark$ & $\checkmark$ & $\checkmark$ \\
\hline Realistic video 1 & $\checkmark$ & $\checkmark$ & $x$ & $\checkmark$ & $\checkmark$ & $x$ & $\checkmark$ & $\checkmark$ & $\checkmark$ & $\checkmark$ & $\checkmark$ & $\checkmark$ & $\checkmark$ & $x$ \\
\hline Realistic video 2 & $x$ & $\times$ & $x$ & $\times$ & $\checkmark$ & $x$ & $\checkmark$ & $\checkmark$ & $\checkmark$ & $\checkmark$ & $\checkmark$ & $\checkmark$ & $x$ & $x$ \\
\hline Cartoon video 1 & $\checkmark$ & $\checkmark$ & $\checkmark$ & $\checkmark$ & $x$ & $\checkmark$ & $\checkmark$ & $\checkmark$ & $x$ & $\checkmark$ & $\checkmark$ & $\checkmark$ & $\checkmark$ & $\checkmark$ \\
\hline Cartoon video 2 & $\checkmark$ & $\checkmark$ & $\times$ & $x$ & $\checkmark$ & $x$ & $x$ & $\checkmark$ & $\times$ & $x$ & $\checkmark$ & $\checkmark$ & $\checkmark$ & $\checkmark$ \\
\hline Human interpreter 1 & $x$ & $x$ & $x$ & $x$ & $x$ & $x$ & $\checkmark$ & $\checkmark$ & $x$ & $x$ & $\checkmark$ & $\checkmark$ & $x$ & $x$ \\
\hline Human interpreter 2 & $x$ & $\checkmark$ & $x$ & $\checkmark$ & $\checkmark$ & $x$ & $x$ & $x$ & $\times$ & $\checkmark$ & $\checkmark$ & $\checkmark$ & $\checkmark$ & $\checkmark$ \\
\hline Animated interpreter 1 & $x$ & $x$ & $x$ & $\times$ & $x$ & $x$ & $x$ & $x$ & $\times$ & $x$ & $x$ & $x$ & $x$ & $x$ \\
\hline Animated interpreter 2 & $x$ & $x$ & $x$ & $x$ & $x$ & $x$ & $x$ & $x$ & $x$ & $x$ & $x$ & $x$ & $x$ & $x$ \\
\hline Hand sign & $x$ & $x$ & $x$ & $\times$ & $x$ & $x$ & $x$ & $x$ & $\times$ & $x$ & $x$ & $\checkmark$ & $\checkmark$ & $x$ \\
\hline Fingerspelling 1 & $\checkmark$ & $\checkmark$ & $x$ & $\checkmark$ & $\checkmark$ & $\checkmark$ & $\checkmark$ & $\checkmark$ & $\checkmark$ & $\checkmark$ & $\checkmark$ & $\checkmark$ & $\checkmark$ & $\checkmark$ \\
\hline Fingerspelling 2 & $x$ & $\checkmark$ & $x$ & $\checkmark$ & $\checkmark$ & $\checkmark$ & $\checkmark$ & $\checkmark$ & $\checkmark$ & $\checkmark$ & $\checkmark$ & $\checkmark$ & $\checkmark$ & $\checkmark$ \\
\hline Fingerspelling 3 & $\checkmark$ & $\checkmark$ & $\checkmark$ & $\checkmark$ & $\checkmark$ & $\checkmark$ & $\checkmark$ & $\checkmark$ & $\checkmark$ & $\checkmark$ & $\checkmark$ & $\checkmark$ & $\checkmark$ & $\checkmark$ \\
\hline
\end{tabular}

Note: (ï) indicates that the participant performed the task correctly, while(') indicates that the participant performed the task incorrectly. 


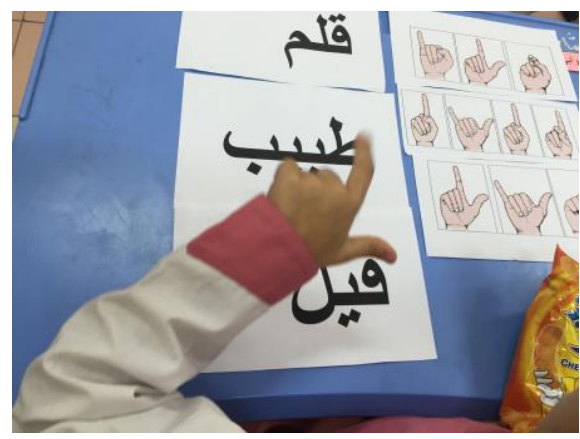

Figure 2. A hearing-impaired child imitating the fingerspelling cards in the text-to-fingerspelling association task.

Text-to-finger-spelling association: Of all the tasks, the children performed the best in the text-to-fingerspelling association task. The children from secondand fifth-grade completed this task successfully for all examples, and only two children from first-grade were unable to complete successfully one of the four associations. There was an interesting observation-during this task, the children imitated fingerspelling images (see Figure 2).

At the end of the tasks, the children applied two AR applications utilising SL and text. Let's Read (Kids App, 2017) interprets stories using ArSL; these stories are also visualised and presented in Arabic text. Magic Camera (Baby Sign and Learn, 2013), in which AR is used to animate an avatar to interpret text accompanied by ASL, was also presented to the children. The older children preferred the storytelling offered by Let's Read, whereas the firstgrade children found Magic Camera more entertaining.

\section{Discussion}

The teachers of elementary school children with hearing impairments who participated in our study advocated the use of ArSL as the main visual representation of printed text in the interviews. The teachers recommended supplementing ArSL with pictures and videos. The parents of the hearingimpaired children (via questionnaires) supported this approach; however, the parents gave higher priority to videos and pictures over ArSL. Interestingly, from the observations of the children, it was evident that the children struggled with ArSL and performed much better in the video and fingerspelling tasks. The majority of the children seemed to also struggle with animated interpreters, highlighting the importance of using real interpreters to 
facilitate understanding. Although the teachers and parents did not consider fingerspelling an important visual medium, the observation results highlighted its utility for demonstrating vocabulary and ArSL. Table 4 summarises the visual representation preferences identified from the user studies.

Table 4

The Visual Needs of Children with Hearing Impairments and the Ranked Preferences for Different Visual Representations as Elicited From Teachers, Parents, and Children

\begin{tabular}{lccc}
\hline \multicolumn{1}{c}{ Visual Medium } & Questionnaire & Interview & Observation \\
\hline Pictures & $\checkmark^{1}$ & $\checkmark^{2}$ & $\checkmark^{2}$ \\
Videos & $\checkmark^{1}$ & $\checkmark^{2}$ & $\checkmark^{3}$ \\
Sign Language (ArSL) & $\checkmark^{2}$ & $\checkmark^{1}$ & $\checkmark^{4}$ \\
Fingerspelling & - & - & $\checkmark^{1}$ \\
\hline
\end{tabular}

Note: The superscript numbers indicate the order of preference.

Word \& Sign is an AR application developed based on the requirements elicited from the hearing-impaired children and their parents and teachers (see Figure 3). An electronic book containing Arabic words extracted from the children's original elementary courseware accompanies this application to supplement their education. The electronic book shows text next to an image representing that text. Because pictures are static in nature, they appear alongside the corresponding text. As the intended users are hearing-impaired children, the designed application supports the decoding process when reading. The application combines pictures, videos, ArSL, and fingerspelling into various options that are easily navigable. The application also provides an audio option for hard-of-hearing children.

The development of the Word \& Sign AR application for Android smartphones used Unity 3D and Vuforia. Due to the lack of open-source ArSL videos, creation of the video resources for ArSL came from the recordings of a 10-year-old hearing-impaired female child interpreter (see Figure 3). When first opened, the application prompts the user to find a target. Upon locating the target, an ArSL video of the corresponding word appears over the image to ensure that the text is always visible to encourage decoding. Several options are available to the user: ArSL with chaining, ArSL with sandwiching, and plain video. 


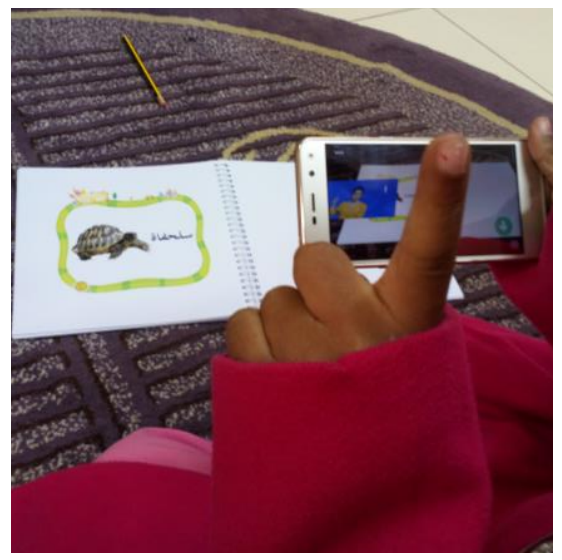

Figure 3. A deaf child using the Word \& Sign application while referring to a book to learn the word "turtle." The child is following a child interpreter as she uses the chaining technique, in which she first fingerspells the word, then uses the ArSL of the word, and finally points to the word.

\section{CONCLUSION AND FUTURE WORK}

The visual needs of Arab children with hearing impairments were previously unaddressed in the literature. In this study, we examined the visual needs of hearing-impaired children from the perspective of the children as well as the perspectives of their parents and teachers. The findings guided the development of an AR application to support the literacy development of such Arab children. The main contribution of this paper is twofold. First, utilising qualitative and quantitative methods to assess and identify the visual needs of children with hearing impairment from the perspectives of the children, their parents and teachers and second, developing an AR application, Word \& Sign to support the literacy development of hearing-impaired Arab children. In the future, we intend to assess the usability of the system with the help of usability experts to refine the system's overall design and evaluate its performance. After this refinement, we intend to conduct experimental studies to determine the application's impact on the literacy skills of school-aged children with hearing impairments.

\section{ACKNOWLEDGMENT}

We would like to thank all participants who took part in this study. We also wish to thank in particular, the directors of Al-Amal Institute, the 300th Public 
Elementary School of Riyadh and the educators for their encouragement and cooperation in the study.

\section{REFERENCES}

Ahmad, W., Nooraishya, W., \& Ali, N. M. (2018). The impact of persuasive technology on user emotional experience and user experience over time. Journal of ICT, 17(4), 601-628.

Al-Nafjan, A., Al-Arifi, B., \& Al-Wabil, A. (2015). Design and development of an educational Arabic sign language mobile application: Collective impact with Tawasol. Proceedings of the International Conference on Universal Access in Human-Computer Interaction (pp. 319-326). https://doi.org/10.1007/978-3-319-20681-3_30

Alnafjan, A., Aljumaah, A., Alaskar, H., \& Alshraihi, R. (2017). Designing "Najeeb": Technology-Enhanced Learning for Children with Impaired Hearing Using Arabic Sign-Language ArSL Applications. Proceedings of the 2017 International Conference on Computer and Applications (pp. 238-273). IEEE. https://doi.org/10.1109/COMAPP.2017.8079772

Alyami, H., Soer, M., Swanepoel, A., \& Pottas, L. (2016). Deaf or hard of hearing children in Saudi Arabia: Status of early intervention services. International Journal of Pediatric Otorhinolaryngology, 86, 142-149. https://doi.org/10.1016/j.ijporl.2016.04.010

Baby Sign and Learn. Magic Camera - American Sign Language Edition. (2018, February 22). Retrieved from https://itunes.apple.com/us/app/ magic-camera-american-sign-language-edition/id662781080? $\mathrm{mt}=8$

Barma, S., Daniel, S., Bacon, N., Gingras, M.-A., \& Fortin, M. (2015). Observation and analysis of a classroom teaching and learning practice based on augmented reality and serious games on mobile platforms. International Journal of Serious Games, 2(2), 69-88. https://doi. org/10.17083/ijsg.v2i2.66

Billinghurst, M., \& Dünser, A. (2012). Augmented reality in the classroom. Computer, 45(7), 56-63. https://doi.org/10.1109/MC.2012.111

Braun, V., \& Clarke, V. (2006). Using thematic analysis in psychology. Using Qualitative Research in Psychology, 3(2), 77-101. https://doi.org/The publisher's URL is: http://dx.doi.org/10.1191/1478088706qp063oa

Cadenanes, J., \& Arrieta, A. G. (2014). Development of Sign Language Communication Skill on Children through augmented reality and the MuCy model. Proceedings of Methodologies and Intelligent Systems for Technology Enhanced Learning (pp. 45-52). https://doi. org/10.1007/978-3-319-07698-0_6

Correa, A. G. D., Tahira, A., \& Ribeir, J. B. (2012). Development of an Interactive Book with Augmented Reality for Teaching and Learning 
Geometric Shapes. Proceedings of the 7th Iberian Conference on Information Systems and Technologies (pp. 1-6).

Craig, A. B., \& Craig, A. B. (2013). Mobile augmented reality. In Understanding Augmented Reality: Concepts and applications (pp. 209-220). Newness. https://doi.org/10.1016/B978-0-240-82408-6.00007-2

DAQRI. Anatomy 4D. (2018, February 20). Retrieved from http://anatomy4d. daqri.com/

Diegmann, P., Schmidt-Kraepelin, M., Van den Eynden, S., Basten, D., Eynden, S. Van Den, \& Basten, D. (2015) Benefits of Augmented Reality in Educational Environments - A Systematic Literature Review. Wirtschaftsinformatik, 3(6), 1542-1556.

Dunleavy, M., Dede, C., \& Mitchell, R. (2009). Affordances and limitations of immersive participatory augmented reality simulations for teaching and learning. Journal of Science Education and Technology, 18(1), 7-22. https://doi.org/10.1007/s10956-008-9119-1

Dünser, A., Grasset, R., \& Billinghurst, M. (2008). A survey of evaluation techniques used in augmented reality studies. New Zealand: Human Interface Technology Laboratory. https://doi. org/10.1145/1508044.1508049

Elkins, J. (2008). The concept of visual literacy, and its limitations. In Visual Literacy (pp. 1-9). Routledge. https://doi.org/10.4324/9780203939574

Garrett, B. M., Jackson, C., \& Wilson, B. (2015). Augmented reality m-learning to enhance nursing skills acquisition in the clinical skills laboratory. Interactive Technology and Smart Education, 12(4), 298-314. https:// doi.org/10.1108/ITSE-05-2015-0013

Geeslin, J. D. (2008). Deaf bilingual education: A comparison of the academic performance of deaf children of deaf parents and deaf children of hearing parents. Bloomington, Indiana: Indiana University.

Georgiou, Y., \& Kyza, E. A. (2017). The development and validation of the ARI questionnaire: An instrument for measuring immersion in location-based augmented reality settings. International Journal of Human Computer Studies, 98, 24-37. https://doi.org/10.1016/j. ijhcs.2016.09.014

Goldin-Meadow, S., Mayberry, R. I., \& Read, T. O. (2001). How Do Profoundly Deaf Children Learn to Read? Learning Disabilities Research \& Practice, 16(4), 222-229. https://doi.org/10.1111/09388982.00022

Hanson, V. L. (1989). Phonology and Reading : Evidence from Profoundly Deaf Readers. In Phonology and Reading Disability: Solving the Reading Puzzle (pp. 67-89). Ann Arbor, Michigan: University of Michigan Press. 
Humphries, T., \& MacDougall, F. (1999). Chaining and other links: Making connections between American Sign Language and English in Two Types of School Settings. Visual Anthropology Review, 15(2), 84-94. https://doi.org/10.1525/var.2000.15.2.84

Kids App. Let's Read. (2017, May 2014). Retrieved from https://itunes.apple. com/us/app//id1205247520? $\mathrm{mt}=8$

Kingdom of Saudi Arabia General Authority for Statistics. Demographic survey. (2017, April 21). Retrieved from https://www.stats.gov.sa/ en $/ 852$

Kožuh, I., Hauptman, S., Kosec, P., \& Debevc, M. (2015). Assessing the efficiency of using augmented reality for learning sign language. Proceedings of the International Conference on Universal Access in Human-Computer Interaction (pp. 404-415). https://doi. org/10.1007/978-3-319-20681-3_38

McIntosh, M. J., \& Morse, J. M. (2015). Situating and Constructing Diversity in Semi-Structured Interviews. Global Qualitative Nursing Research, 2, 1-12. https://doi.org/10.1177/2333393615597674

Mellon, N. K., Niparko, J. K., Rathmann, C., Mathur, G., Humphries, T., Jo Napoli, D., ... Lantos, J. D. (2015). Should All Deaf Children Learn Sign Language? Pediatrics, 136(1), 170-176. https://doi.org/10.1542/ peds.2014-1632

mHealth Company. Hayaak. (2018, January 22). Retrieved from https://itunes. apple.com/gb/app/hayaak/id1152564831? $\mathrm{mt}=8$

Mich, O. (2009). Evaluation of software tools with deaf children. Proceeding of the eleventh international ACM SIGACCESS conference on Computers and accessibility (pp. 235-236). https://doi. org/10.1145/1639642.1639692

Mind Rockets Inc. Alturjuman. (2018, January 19). Retrieved from https://play. google.com/store/apps/details?id=com.mindrockets.turjoman\&hl=ar

Nowell, L. S., Norris, J. M., White, D. E., \& Moules, N. J. (2017). Thematic Analysis: Striving to Meet the Trustworthiness Criteria. International Journal of Qualitative Methods, 16(1), 1-13. https://doi. org/10.1177/1609406917733847

O'Shea, P. M., Dede, C., \& Cherian, M. (2011). The Results of Formatively Evaluating an Augmented Reality Curriculum Based on Modified Design Principles. International Journal of Gaming and Computer-Mediated Simulations, 3(2), 57-66. https://doi.org/10.4018/jgcms.2011040104

Oka Sudana, A. A. K., Aristamy, I. G. A. A. M., \& Wirdiani, N. K. A. (2016). Augmented reality application of sign language for deaf people in Android based on smartphone. International Journal of Software Engineering and Its Applications, 10(8), 139-150. https://doi. org/10.14257/ijseia.2016.10.8.13

Pinto, D., Mosquera, J., Gonzalez, C., Tobar-Muñoz, H., Baldiris, S., \& Fabregat, R. (2017). Augmented Reality Board Game for supporting 
learning and motivation in an indigenous community. Proceedings of the V International Congress on Videogames \& Education (pp. 213222).

Samuel, R. J., \& Zaitun, A. B. (2007). Do Teachers have Adequate ICT Resources and the Right ICT Skills in Integrating ICT Tools in the Teaching and Learning of English Language in Malaysian Schools? The Electronic Journal of Information Systems in Developing Countries, 29(1), 1-15. https://doi.org/10.1002/j.1681-4835.2007.tb00196.x

Sungkur, R. K., Panchoo, A., \& Bhoyroo, N. K. (2016). Augmented reality, the future of contextual mobile learning. Interactive Technology and Smart Education, 13(2), 123-146. https://doi.org/10.1108/ITSE-072015-0017

Treiman, R., \& Hirsh-Pasek, K. (1983). Silent reading: Insights from secondgeneration deaf readers. Cognitive Psychology, 15(1), 39-65. https:// doi.org/10.1016/0010-0285(83)90003-8

Turner, D. W. (2010). Qualitative interview design: A practical guide for novice investigators. The Qualitative Report, 15(3), 754-760. https:// doi.org/http://www.nova.edu/ssss/QR/QR15-3/qid.pdf

Uluyol, Ç., \& Sahin, S. (2016). Augmented reality: A new direction in education. In Emerging Tools and Applications of Virtual Reality in Education (pp. 239-257). IGI Global. https://doi.org/10.4018/978-14666-9837-6.ch011

Wojciechowski, R., \& Cellary, W. (2013). Evaluation of learners' attitude toward learning in ARIES augmented reality environments. Computers and Education, 68, 570-585. https://doi.org/10.1016/j. compedu.2013.02.014

Xu, Y., Spasojevic, M., Gao, J., \& Jacob, M. (2008). Designing a vision-based mobile interface for in-store shopping. Proceedings of the 5th Nordic conference on Human-computer interaction (pp. 393-402). https://doi. org/10.1145/1463160.1463203

Yilmaz, R. M. (2016). Educational magic toys developed with augmented reality technology for early childhood education. Computers in Human Behavior, 54, 240-248. https://doi.org/10.1016/j.chb.2015.07.040

Zainuddin, N. M. M., Zaman, H. B., \& Ahmad, A. (2009). Learning science using AR book: A preliminary study on visual needs of deaf learners. Proceedings of the International Visual Informatics Conference (pp. 844-855). https://doi.org/10.1007/978-3-642-05036-7_80

Zainuddin, N. M. M., Zaman, H. B., \& Ahmad, A. (2011). Heuristic evaluation on Augmented Reality courseware for the deaf. Proceedings of the International Conference on User Science and Engineering (pp. 183188). https://doi.org/10.1109/iUSEr.2011.6150562

Zhu, E., Hadadgar, A., Masiello, I., \& Zary, N. (2014). Augmented reality in healthcare education: an integrative review. PeerJ, 2, e469. https://doi. org/10.7717/peerj.469 